\title{
On a $k$-th Gauduchon almost Hermitian manifold
}

https://doi.org/10.1515/coma-2021-0130

Received October 18, 2021; accepted January 22, 2022

\begin{abstract}
We characterize the $k$-th Gauduchon condition and by applying its characterization, we reprove that a compact $k$-th Gauduchon, semi-Kähler manifold becomes quasi-Kähler, which tells us that in particular, a compact almost pluriclosed, semi-Kähler manifold is quasi-Kähler.
\end{abstract}

Keywords: almost Hermitian manifold, $k$-th Gauduchon metric, Chern connection

MSC: $32 \mathrm{Q} 60$ (primary); 53C15; 53C55 (secondary)

\section{Introduction}

In [3], J. Fu, Z. Wang and D. Wu introduced the $k$-th Gauduchon metric as a generalized version of the Gauduchon metric. From the definition, we see that $(n-1)$-th Gauduchon metrics are the usual Gauduchon metrics, and astheno-Kähler metrics (cf. [8]) are examples of $(n-2)$-th Gauduchon metrics, pluriclosed metrics are 1-st Gauduchon metrics. A. Fino and L. Ugarte have shown that for each $k=1, \ldots,\left[\frac{n}{2}\right]-1$, a Hermitian metric is $k$-th Gauduchon if and only if it is $(n-k-1)$-th Gauduchon on a complex nilmanifold (cf. [2, Lemma 4.7]). A. Latorre and L. Ugarte have investigated the $k$-th Gauduchon condition on homogeneous compact complex manifolds (cf. [12]). In [1], H. Chen, L. Chen and X. Nie investigated the $k$-th Gauduchon metric and characterized it on a compact Hermitian manifold. They also obtained a direct corollary that if the manifold is $k$-th Gauduchon, then one has $s \geq \hat{s}$, where $s$ is the Chern scalar curvature and $\hat{s}$ is the Riemannian type scalar curvature. We extend thier characterization result to almost Hermitian geometry. We have already defined the $k$-th Gauduchon metric on almost Hermitian manifolds and have obtained some results in $[10,11]$.

Let $\left(M^{2 n}, J\right)$ be an almost complex manifold of real dimension $2 n$ with $n \geq 3$ and let $g$ be an almost Hermitian metric on $M$. Let $\left\{Z_{r}\right\}$ be an arbitrary local $(1,0)$-frame around a fixed point $p \in M$ and let $\left\{\zeta^{r}\right\}$ be the associated coframe. Then the associated real $(1,1)$-form $\omega$ with respect to $g$ takes the local expression $\omega=\sqrt{-1} g_{r \bar{k}} \zeta^{r} \wedge \zeta^{\bar{k}}$. We will also refer to $\omega$ as to an almost Hermitian metric in the present paper. We define a Gauduchon metric and a $k$-th Gauduchon metric on an almost Hermitian manifold in the following.

Definition 1.1. Let $\left(M^{2 n}, J\right)$ be an almost complex manifold. An almost Hermitian metric $\omega$ is called a Gauduchon metric on $M$ if $\omega$ satisfies $d^{\star}\left(J d^{*} \omega\right)=0$, where $d^{\star}$ is the adjoint of $d$ with respect to the metric $\omega$, which is equivalent to that $d\left(J d\left(\omega^{n-1}\right)\right)=0$, also equivalent to that $\partial \bar{\partial}\left(\omega^{n-1}\right)=0$. When an almost Hermitian metric $\omega$ is Gauduchon, we call the triple $\left(M^{2 n}, J, \omega\right)$ a Gauduchon almost Hermitian manifold. For an integer $k$ such that $1 \leq k \leq n-1$, an almost Hermitian metric $\omega$ is called $k$-th Gauduchon if the metric $\omega$ satisfies that

$$
\partial \bar{\partial} \omega^{k} \wedge \omega^{n-k-1}=0 .
$$

When an almost Hermitian metric $\omega$ is $k$-th Gauduchon, we call the triple $\left(M^{2 n}, J, \omega\right)$ a $k$-th Gauduchon almost Hermitian manifold. *Corresponding Author: Masaya Kawamura: Department of General Education, National Institute of Technology, Kagawa
College, 355, Chokushi-cho, Takamatsu, Kagawa, Japan 761-8058, E-mail: kawamura-m@t.kagawa-nct.ac.jp 
Note that in the case of $n=2$, a Gauduchon metric and a $k$-th Gauduchon metric are both almost pluriclosed (see Definition 1.3).

One has the following well-known result.

Proposition 1.1. (cf. [4]) Let $\left(M^{2 n}, J, \omega\right)$ be a compact almost Hermitian manifold with $n \geq 2$. Then there exists a smooth function $u$, unique up to addition of a constant, such that the conformal almost Hermitian metric $e^{u} \omega$ is Gauduchon.

We characterize the $k$-th Gauduchon condition on a compact almost Hermitian manifold as follows.

Theorem 1.1. Let $\left(M^{2 n}, J, \omega\right)$ be compact almost Hermitian manifold with $n \geq 3$ and let $k$ be an integer such that $1 \leq k \leq n-1$. Then the following are equivalent.

(i) $\omega$ is $k$-th Gauduchon;

(ii) $s-\hat{s}=\frac{k-1}{n-2}\left|\partial^{\star} \omega\right|^{2}+\frac{n-k-1}{n-2}|\partial \omega|^{2}+B_{i j}^{\bar{r}} B_{\bar{r} \bar{j}}^{i}$,

where $s$ is the Chern scalar curvature and $\hat{s}$ is a Riemannian type scalar curvature of the metric $\omega$ with respect to the Chern connection (see (2.10)), and $B_{i j}^{\tilde{r}}$ 's are the structure coefficients of Lie bracket (see (2.7), (2.8) for more detail). Note that $B_{i j}^{\bar{r}} B_{\bar{r} \bar{j}}^{i}$ means that we sum over repeated indices $i, j$ and $r$ with respect to the metric $\omega$.

Remark 1.1. On a $k$-th Gauduchon almost Hermitian manifold, if $B_{i j}^{\bar{r}} B_{\bar{r} \bar{j}}^{i} \geq 0$, then we have that $s \geq \hat{s}$ from Theorem 1.1. The condition $B_{i j}^{\bar{r}} B_{\bar{r} \bar{j}}^{i} \geq 0$ also appears in other situations. For instance, on a Kähler-like and GKähler-like almost Hermitian manifold $\left(M^{2 n}, J, \omega\right)$ with $n \geq 2$, if $B_{i j}^{\bar{r}} B_{\bar{r} \bar{j}}^{i} \geq 0$, then the metric $\omega$ is semi-Kähler (see Definition 1.2) and the almost complex structure $J$ is integrable (i.e., the metric $\omega$ is balanced) (cf. [9, Corollary 1.2]).

We define a semi-Kähler metric on almost complex manifolds. Note that when a manifold is complex, a semiKähler metric is called a balanced metric.

Definition 1.2. (cf. [6]) Let $\left(M^{2 n}, J\right)$ be an almost complex manifold. An almost Hermitian metric $\omega$ is called semi-Kähler if the metric $\omega$ satisfies $d\left(\omega^{n-1}\right)=0$. When an almost Hermitian metric $\omega$ is semi-Kähler, the triple $\left(M^{2 n}, J, \omega\right)$ is called a semi-Kähler manifold.

Recall that a quasi-Kähler structure $(J, \omega)$ on a smooth differentiable manifold $M$ is an almost Hermitian structure whose associated metric $\omega$ satisfies that

$$
(d \omega)^{(1,2)}=\bar{\partial} \omega=0,
$$

which is equivalent to the original definition of quasi-Kählerity:

$$
D_{X} J(Y)+D_{J X} J(J Y)=0
$$

for all vector fields $X, Y$, where $D$ denotes the Levi-Civita connection (cf. [6]). An almost Hermitian metric $\omega$ is called quasi-Kähler if the metric $\omega$ satisfies (1.1). When an almost Hermitian metric $\omega$ is quasi-Kähler, the triple $(M, J, \omega)$ is called a quasi-Kähler manifold.

K. Liu and X. Yang have shown that if a compact $k$-th Gauduchon Hermitian manifold is also balanced, then it is Kähler in [13].

Proposition 1.2. (cf. [13, Corollary 1.16]) Let $(M, J, \omega)$ be a compact $k$-th Gauduchon, balanced manifold with $n \geq 3$ and let $k$ be an integer such that $1 \leq k \leq n-2$. Then $\omega$ is Kähler.

By applying the characterization of the $k$-th Gauduchon condition in almost Hermitian geometry, we have the following generalized result of Proposition 1.2. This has actually been already proven in a different way in [11, Theorem 1.1]. 
Corollary 1.1. Let $\left(M^{2 n}, J, \omega\right)$ be a compact $k$-th Gauduchon, semi-Kähler manifold with $n \geq 3$ and let $k$ be an integer such that $1 \leq k \leq n-2$. Then $\omega$ is quasi-Kähler.

We define an almost pluriclosed metric on almost complex manifolds. Note that in the case of complex manifolds, an almost pluriclosed metric is called a pluriclosed metric.

Definition 1.3. Let $(M, J)$ be an almost complex manifold. An almost Hermitian metric $\omega$ is called almost pluriclosed if the metric $\omega$ satisfies that $\partial \bar{\partial} \omega=0$. When an almost Hermitian metric $\omega$ is almost pluriclosed, the triple $(M, J, \omega)$ is called an almost pluriclosed manifold.

We introduce the following well-known result in Hermitian geometry.

Proposition 1.3. (cf. [14, Proposition 1.1]) Let $(M, J, \omega)$ be a compact Hermitian manifold. If the metric $\omega$ is pluriclosed and balanced, then $\omega$ is Kähler.

We can show the following generalized version of Proposition 1.3 in almost Hermitian geometry by applying Corollary 1.1, since an almost pluriclosed metric is 1-st Gauduchon. Note that when the real dimension of an almost Hermitian manifold is four (i.e., $n=2$ ), a semi-Käler metric becomes quasi-Kähler since a semi-Kähler metric is almost Kähler for $n=2$, and in general, an almost Kähler metric is quasi-Kähler (cf. [7, Theorem 4.2]).

Corollary 1.2. Let $(M, J, \omega)$ be a compact almost Hermitian manifold. If the metric $\omega$ is almost pluriclosed and semi-Kähler, then $\omega$ is quasi-Kähler.

This paper is organized as follows: in the second section, we recall some basic definitions and computations in almost Hermitian geometry. In the third section, we give a proof of main result and by applying it, we show some corollaries. Notice that we assume the Einstein convention omitting the symbol of sum over repeated indices in all this paper.

\section{Preliminaries}

\subsection{The bundle of real $k$-forms and the interior product}

Let $M$ be a real $2 n$-dimensional smooth differentiable manifold and let $h$ be a Riemannian metric on $M$. In a local coordinate $\left(x^{1}, x^{2}, \ldots, x^{2 n}\right)$ on $M$, we write $h=h_{i j} d x^{i} d x^{j}$. Denote $\left(h^{i j}\right)$ the inverse matrix of $\left(h_{i j}\right)$, $1 \leq i, j \leq 2 n$. Then the metric $h$ induces an inner product $\langle\cdot, \cdot \cdot\rangle$ on the cotangent bundle $T^{*} M$ by $\left\langle d x^{i}, d x^{j}\right\rangle=h^{i j}$. Let $\Lambda^{k} T^{\star} M$ be the bundle of real $k$-forms for $1 \leq k \leq 2 n$. The inner product induced by $h$ on $\Lambda^{k} T^{\star} M$ is given by

$$
\left\langle\alpha_{1} \wedge \cdots \wedge \alpha_{k}, \beta_{1} \wedge \cdots \wedge \beta_{k}\right\rangle=\operatorname{det}\left(\left\langle\alpha_{i}, \beta_{j}\right\rangle\right),
$$

for $\alpha_{i}, \beta_{j} \in T^{\star} M$. For $\varphi=\frac{1}{k !} \varphi_{i_{1} \ldots i_{k}} d x^{i_{1}} \wedge \cdots \wedge d x^{i_{k}}, \psi=\frac{1}{k !} \psi_{j_{1} \ldots j_{k}} d x^{j_{1}} \wedge \cdots \wedge d x^{j_{k}}$, where $\varphi_{i_{1} \ldots i_{k}}$ is skew symmetric in $i_{1}, \ldots, i_{k}$ and $\psi_{j_{1} \ldots j_{k}}$ is skew symmetric in $j_{1}, \ldots, j_{k}$,

$$
\langle\varphi, \psi\rangle=\frac{1}{k !} h^{i_{1} j_{1}} \cdots h^{i_{k} j_{k}} \varphi_{i_{1} \ldots i_{k}} \psi_{j_{1} \ldots j_{k}} .
$$

We define the interior product $\iota_{X} \varphi \in \Lambda^{k-1} T^{\star} M$ for vector fields $X, X_{1}, \ldots, X_{k-1}$ on $M$ and $\varphi \in \Lambda^{k} T^{\star} M$ by

$$
\iota_{X} \varphi\left(X_{1}, \ldots, X_{k-1}\right):=\varphi\left(X, X_{1}, \ldots, X_{k-1}\right) .
$$

Note that we have

$$
\iota_{X}\left(\alpha_{1} \wedge \cdots \wedge \alpha_{k}\right)=\sum_{i=1}^{k}(-1)^{i-1} \alpha_{i}(X) \alpha_{1} \wedge \cdots \wedge \alpha_{i-1} \wedge \widehat{\alpha}_{i} \wedge \alpha_{i+1} \wedge \cdots \wedge \alpha_{k} .
$$


Define $\tilde{X}:=h(X, \cdot) \in T^{\star} M$, then we obtain that for $\varphi \in \Lambda^{k+1} T^{\star} M$ and $\psi \in \Lambda^{k} T^{\star} M$ (cf. [1, (2.3)]),

$$
\left\langle\iota_{X} \varphi, \psi\right\rangle=\langle\varphi, \tilde{X} \wedge \psi\rangle .
$$

\subsection{The Nijenhuis tensor of the almost complex structure}

An almost complex structure on $M$ is an endomorphism $J$ of $T M, J \in \Gamma(\operatorname{End}(T M))$, satisfying $J^{2}=-I d_{T M}$, where $T M$ is the real tangent vector bundle of $M$. The pair $(M, J)$ is called an almost complex manifold. Let $(M, J)$ be an almost complex manifold. We define a bilinear map on $C^{\infty}(M)$ for $X, Y \in \Gamma(T M)$ by

$$
4 N(X, Y):=[J X, J Y]-J[J X, Y]-J[X, J Y]-[X, Y],
$$

which is the Nijenhuis tensor of $J$. The Nijenhuis tensor $N$ satisfies $N(X, Y)=-N(Y, X), N(J X, Y)=-J N(X, Y)$, $N(X, J Y)=-J N(X, Y), N(J X, J Y)=-N(X, Y)$. For any $(1,0)$-vector fields $W$ and $V, N(V, W)=-[V, W]^{(0,1)}$, $N(V, \bar{W})=N(\bar{V}, W)=0$ and $N(\bar{V}, \bar{W})=-[\bar{V}, \bar{W}]^{(1,0)}$ since we have $4 N(V, W)=-2([V, W]+\sqrt{-1} J[V, W])$, $4 N(\bar{V}, \bar{W})=-2([\bar{V}, \bar{W}]-\sqrt{-1} J[\bar{V}, \bar{W}])$. An almost complex structure $J$ is called integrable if $N=0$ everywhere on $M$. Giving a complex structure on a smooth differentiable manifold $M$ is equivalent to giving an integrable almost complex structure on $M$.

Let $(M, J)$ be an almost complex manifold. A Riemannian metric $h$ on $M$ is called $J$-invariant if $J$ is compatible with $h$, i.e., for any $X, Y \in \Gamma(T M), h(X, Y)=h(J X, J Y)$. In this case, the pair $(J, h)$ is called an almost Hermitian structure. The fundamental 2-form $\omega$ associated to a $J$-invariant Riemannian metric $h$, i.e., an almost Hermitian metric, is determined by, for $X, Y \in \Gamma(T M), \omega(X, Y)=h(J X, Y), \omega \in \Gamma\left(\bigwedge^{2} T^{\star} M\right)$.

The complexified tangent vector bundle is given by $T^{\mathbb{C}} M=T M \otimes_{\mathbb{R}} \mathbb{C}$ for the real tangent vector bundle $T M$. By extending $J \mathbb{C}$-linearly and $g, \mathbb{C}$-bilinearly to $T^{\mathbb{C}} M$, they are also defined on $T^{\mathbb{C}} M$ and we observe that the complexified tangent vector bundle $T^{\mathbb{C}} M$ can be decomposed as $T^{\mathbb{C}} M=T^{1,0} M \oplus T^{0,1} M$, where $T^{1,0} M$, $T^{0,1} M$ are the eigenspaces of $J$ corresponding to eigenvalues $\sqrt{-1}$ and $-\sqrt{-1}$, respectively:

$$
T^{1,0} M=\{X-\sqrt{-1} J X \mid X \in T M\}, \quad T^{0,1} M=\{X+\sqrt{-1} J X \mid X \in T M\} .
$$

Let $\Lambda^{r} M=\bigoplus_{p+q=r} \Lambda^{p, q} M$ for $0 \leq r \leq 2 n$ denote the decomposition of complex differential $r$-forms into $(p, q)$-forms, where

$$
\begin{gathered}
\Lambda^{p, q} M:=\Lambda^{p}\left(\Lambda^{1,0} M\right) \otimes \Lambda^{q}\left(\Lambda^{0,1} M\right), \\
\Lambda^{1,0} M:=\left\{\alpha+\sqrt{-1} J \alpha \mid \alpha \in \Lambda^{1} M\right\}, \quad \Lambda^{0,1} M:=\left\{\alpha-\sqrt{-1} J \alpha \mid \alpha \in \Lambda^{1} M\right\}
\end{gathered}
$$

and $\Lambda^{1} M$ denotes the dual of $T^{\mathbb{C}} M$.

Let $\left\{Z_{r}\right\}$ be a local $(1,0)$-frame with respect to an almost Hermitian metric $g$ and let $\left\{\zeta^{r}\right\}$ be a local associated coframe with respect to $\left\{Z_{r}\right\}$, i.e., $\zeta^{i}\left(Z_{j}\right)=\delta_{j}^{i}$ for $i, j=1, \ldots, n$. We write $g_{i \bar{j}}:=g\left(Z_{i}, Z_{\bar{j}}\right)$. The fundamental $(1,1)$-form $\omega$ associated to $g$ is locally given by $\omega=\sqrt{-1} g_{i j} \zeta^{i} \wedge \zeta^{\bar{j}}$. Since $g$ is almost Hermitian, its components satsfy $g_{i j}=g_{\overline{i j}}=0$ and $g_{i \bar{j}}=g_{\overline{j i}}=\bar{g}_{\overline{i j}}$. By using these local frame $\left\{Z_{r}\right\}$ and coframe $\left\{\zeta^{r}\right\}$, we have

$$
N\left(Z_{\bar{i}}, Z_{\bar{j}}\right)=-\left[Z_{\bar{i}}, Z_{\bar{j}}\right]^{(1,0)}=: N_{\bar{i} j}^{k} Z_{k}, \quad N\left(Z_{i}, Z_{j}\right)=-\left[Z_{i}, Z_{j}\right]^{(0,1)}=\overline{N_{\bar{i} j}^{k}} Z_{\bar{k}},
$$

and

$$
N=\frac{1}{2} \overline{N_{\bar{i}}^{k}} Z_{\bar{k}} \otimes\left(\zeta^{i} \wedge \zeta^{j}\right)+\frac{1}{2} N_{\overline{i j}}^{k} Z_{k} \otimes\left(\zeta^{\bar{i}} \wedge \zeta^{\bar{j}}\right) .
$$

Let $\left(M^{2 n}, J, g\right)$ be an almost Hermitian manifold. An affine connection $D$ on $T^{\mathbb{C}} M$ is called almost Hermitian connection if $D g=D J=0$. For the almost Hermitian connection, we have the following Lemma (cf. [5], [15], [18]).

Lemma 2.1. Let $(M, J, g)$ be an almost Hermitian manifold with $\operatorname{dim}_{\mathbb{R}} M=2 n$. Then for any given vector valued $(1,1)$-form $\Theta=\left(\Theta^{i}\right)_{1 \leq i \leq n}$, there exists a unique almost Hermitian connection $D$ on $(M, J, g)$ such that the $(1,1)$-part of the torsion is equal to the given $\Theta$. 
If the $(1,1)$-part of the torsion of an almost Hermitian connection vanishes everywhere, then the connction is called the second canonical connection or the Chern connection. We will refer the connection as the Chern connection and denote it by $\nabla$.

Now let $\nabla$ be the Chern connection on $M$. We denote the structure coefficients of Lie bracket by

$$
\begin{gathered}
{\left[Z_{i}, Z_{j}\right]=: B_{i j}^{r} Z_{r}+B_{i j}^{\bar{r}} Z_{\bar{r}}=B_{i j}^{r} Z_{r}-\overline{N_{i j}^{r}} Z_{\bar{r}}, \quad\left[Z_{i}, Z_{\bar{j}}\right]=: B_{i j}^{r} Z_{r}+B_{i j}^{\bar{r}} Z_{\bar{r}},} \\
{\left[Z_{\bar{i}}, Z_{\bar{j}}\right]=: B_{i \bar{j}}^{r} Z_{r}+B_{i \bar{j}}^{\bar{r}} Z_{\bar{r}}=-N_{i \bar{j}}^{r} Z_{r}+B_{i \bar{i}}^{\bar{r}} Z_{\bar{r}},}
\end{gathered}
$$

where we used that $\left[Z_{i}, Z_{j}\right]^{(0,1)}=-\overline{N_{\bar{i}}^{r}} Z_{\bar{r}},\left[Z_{\bar{i}}, Z_{\bar{j}}\right]^{(1,0)}=-N_{i \bar{j}}^{r} Z_{r}$ and then $B_{i j}^{\bar{r}}=-\overline{N_{\bar{i}}^{r}}, B_{\bar{i} j}^{r}=-N_{\bar{i} j}^{r}$. Notice that $J$ is integrable if and only if the $B_{i j}^{\bar{r}}$ 's vanish.

\subsection{The torsion and the curvature on almost complex manifolds}

Since the Chern connection $\nabla$ preserves $J$, we have

$$
\nabla_{i} Z_{j}=\Gamma_{i j}^{r} Z_{r}, \quad \nabla_{i} Z_{\bar{j}}=\Gamma_{i \bar{j}}^{\bar{r}} Z_{\bar{r}},
$$

where $\Gamma_{i j}^{r}=g^{r \bar{s}} Z_{i}\left(g_{j \bar{s}}\right)-g^{r \bar{s}} g_{j \bar{l}} B_{i \bar{s}}^{\bar{l}}$. We can obtain that

$$
\Gamma_{i \bar{j}}^{\bar{r}}=B_{i \bar{j}}^{\bar{r}}
$$

since the $(1,1)$-part of the torsion of the Chern connection vanishes everywhere. Note that the mixed derivatives $\nabla_{i} Z_{\bar{j}}$ do not depend on $g$ (cf. [15]).

Let $\left\{\gamma_{j}^{i}\right\}$ be the connection form, which is defined by $\gamma_{j}^{i}=\Gamma_{s j}^{i} \zeta^{s}+\Gamma_{\bar{s} j}^{i} \zeta^{\bar{s}}$. The torsion $T$ of the Chern connection $\nabla$ is given by $T^{i}=d \zeta^{i}-\zeta^{p} \wedge \gamma_{p}^{i}, T^{\bar{i}}=d \zeta^{\bar{i}}-\zeta^{\bar{p}} \wedge \gamma_{\bar{p}}^{\bar{i}}$, which has no $(1,1)$-part and the only non-vanishing components are as follows:

$$
T_{i j}^{S}=\Gamma_{i j}^{S}-\Gamma_{j i}^{S}-B_{i j}^{S}, \quad T_{i j}^{\bar{S}}=N_{i j}^{\bar{S}}=-B_{i j}^{\bar{S}} .
$$

These tell us that $T=\left(T^{i}\right)$ splits into $T=T^{\prime}+T^{\prime \prime}$, where $T^{\prime} \in \Gamma\left(\Lambda^{2,0} M \otimes T^{1,0} M\right), T^{\prime \prime} \in \Gamma\left(\Lambda^{0,2} M \otimes T^{1,0} M\right)$. We lower the index of torsion and denote it by

$$
T_{i j \bar{k}}=T_{i j}^{S} g_{s \bar{k}} .
$$

Note that $T^{\prime \prime}$ depends only on $J$ and it can be regarded as the Nijenhuis tensor of $J$, that is, $J$ is integrable if and only if $T^{\prime \prime}$ vanishes.

We denote by $\Omega$ the curvature of the Chern connection $\nabla$. We can regard $\Omega$ as a section of $\Lambda^{2} M \otimes \Lambda^{1,1} M$, $\Omega \in \Gamma\left(\Lambda^{2} M \otimes \Lambda^{1,1} M\right)$ and $\Omega$ splits in $\Omega=H+R+\bar{H}$, where $R \in \Gamma\left(\Lambda^{1,1} M \otimes \Lambda^{1,1} M\right), H \in \Gamma\left(\Lambda^{2,0} M \otimes \Lambda^{1,1} M\right)$. The curvature form can be expressed by $\Omega_{j}^{i}=d \gamma_{j}^{i}+\gamma_{s}^{i} \wedge \gamma_{j}^{s}$.

In terms of $Z_{r}$ 's, we have

$$
\begin{aligned}
& R_{i \bar{j} k}{ }^{r}=\Omega_{k}^{r}\left(Z_{i}, Z_{\bar{j}}\right)=Z_{i}\left(\Gamma_{\bar{j} k}^{r}\right)-Z_{\bar{j}}\left(\Gamma_{i k}^{r}\right)+\Gamma_{i s}^{r} \Gamma_{\bar{j} k}^{s}-\Gamma_{\bar{j} s}^{r} \Gamma_{i k}^{s}-B_{i j}^{s} \Gamma_{s k}^{r}+B_{\bar{j} i}^{\bar{s}} \Gamma_{\bar{s} k}^{r}=-R_{\overline{j i k}}{ }^{r}, \\
& H_{i j k}{ }^{r}=\Omega_{k}^{r}\left(Z_{i}, Z_{j}\right)=Z_{i}\left(\Gamma_{j k}^{r}\right)-Z_{j}\left(\Gamma_{i k}^{r}\right)+\Gamma_{i s}^{r} \Gamma_{j k}^{s}-\Gamma_{j s}^{r} \Gamma_{i k}^{s}-B_{i j}^{s} \Gamma_{s k}^{r}-B_{i j}^{\bar{s}} \Gamma_{\bar{s} k}^{r}=-H_{j i k}{ }^{r}, \\
& H_{\overline{i j} k}{ }^{r}=\Omega_{k}^{r}\left(Z_{\bar{i}}, Z_{\bar{j}}\right)=Z_{\bar{i}}\left(\Gamma_{\bar{j} k}^{r}\right)-Z_{\bar{j}}\left(\Gamma_{\bar{i} k}^{r}\right)+\Gamma_{\bar{i} s}^{r} \Gamma_{\bar{j} k}^{s}-\Gamma_{\bar{j} s}^{r} \Gamma_{\bar{i} k}^{s}-B_{i j}^{s} \Gamma_{s k}^{r}-B_{\bar{i} j}^{\bar{s}} \Gamma_{\bar{s} k}^{r}=-H_{\overline{j i k} k}{ }^{r} .
\end{aligned}
$$

We can write $\Omega=\left(\Omega_{j}^{i}\right)=\Omega^{(2,0)}+\Omega^{(1,1)}+\Omega^{(0,2)}=H+R+\bar{H}$, with

$$
\Omega^{(2,0)}=\left(\frac{1}{2} H_{i j k}{ }^{r} \zeta^{i} \wedge \zeta^{j}\right), \quad \Omega^{(1,1)}=\left(R_{i \bar{j} k}^{r} \zeta^{i} \wedge \zeta^{\bar{j}}\right), \quad \Omega^{(0,2)}=\left(\frac{1}{2} H_{\overline{i j k} k}{ }^{r} \zeta^{\bar{i}} \wedge \zeta^{\bar{j}}\right) .
$$

We deduce that

$$
P_{i \bar{j}}=g^{k \bar{l}} R_{i \bar{j} k \bar{l}}, \quad S_{i \bar{j}}=g^{k \bar{l}} R_{k \bar{l} i \bar{j}} \quad R_{i j}=g^{k \bar{l}} H_{i j k \bar{l}}, \quad R_{\bar{i} \bar{j}}=g^{k \bar{l}} H_{\bar{i} \bar{j} k \bar{l}} .
$$

We define the Chern scalar curvature $s$ and a Riemannian type scalar curvature $\hat{s}$ of the metric $\omega$ with respect to the Chern connection:

$$
s:=g^{i \bar{j}} g^{k \bar{l}} R_{i \bar{j} k \bar{l}}=g^{i \bar{j}} P_{i \bar{j}}=g^{i \bar{j}} S_{i \bar{j}}, \quad \hat{s}:=g^{i \bar{l}} g^{k \bar{j}} R_{i \bar{j} k \bar{l}}
$$


Lemma 2.2. (The first Bianchi identity for the Chern curvature) For any $X, Y, Z \in T^{\mathbb{C}} M$,

$$
\sum \Omega(X, Y) Z=\sum\left(T(T(X, Y), Z)+\nabla_{X} T(Y, Z)\right),
$$

where the sum is taken over all cyclic permutations.

This identity induces the following formula:

$$
R_{i \bar{j} k}{ }^{l}=R_{k \overline{j i}}{ }^{l}-T_{i k}^{\bar{r}} T_{\bar{r} \bar{j}}^{l}+\nabla_{\bar{j}} T_{k i}^{l}=R_{k \overline{j i}}{ }^{l}-B_{i k}^{\bar{r}} B_{\bar{r} \bar{j}}^{l}+\nabla_{\bar{j}} T_{k i}^{l},
$$

where used that $R_{i j \bar{k} \bar{l}}=R_{\overline{i j} k l}=0$.

\subsection{The exterior differential operator}

Note that for any $p$-form $\psi$, there holds that

$$
\begin{aligned}
d \psi\left(X_{1}, \ldots, X_{p+1}\right)= & \sum_{i=1}^{p+1}(-1)^{i+1} X_{i}\left(\psi\left(X_{1}, \ldots, \widehat{X}_{i}, \ldots, X_{p+1}\right)\right) \\
& +\sum_{i<j}(-1)^{i+j} \psi\left(\left[X_{i}, X_{j}\right], X_{1}, \ldots, \widehat{X}_{i}, \ldots, \widehat{X}_{j}, \ldots, X_{p+1}\right)
\end{aligned}
$$

for any vector fields $X_{1}, \ldots, X_{p+1}$ on $M$ (cf. [18]). We directly compute that

$$
d \zeta^{s}=-\frac{1}{2} B_{k l}^{s} \zeta^{k} \wedge \zeta^{l}-B_{k l}^{s} \zeta^{k} \wedge \zeta^{\bar{l}}+\frac{1}{2} N_{\bar{k} l}^{s} \zeta^{\bar{k}} \wedge \zeta^{\bar{l}} .
$$

For any $(0,1)$-form $\beta$, we have that $\partial \beta=\partial_{k} \beta_{\bar{j}} \zeta^{k} \wedge \zeta^{\bar{j}}$, and from (2.10) and (2.13),

$$
\nabla_{k} \beta_{\bar{j}}=Z_{k}\left(\beta_{\bar{j}}\right)-\Gamma_{k j}^{\bar{l}} \beta_{\bar{l}}=Z_{k}\left(\beta_{\bar{j}}\right)-B_{k \bar{j}}^{\bar{l}} \beta_{\bar{l}}=\partial_{k} \beta_{\bar{j}} .
$$

For any real $(1,1)$-form $\sigma=\sqrt{-1} \sigma_{i j} \zeta^{i} \wedge \zeta^{\bar{j}}$, we have

$$
\partial \sigma=\frac{\sqrt{-1}}{2}\left(Z_{i}\left(\sigma_{j \bar{k}}\right)-Z_{j}\left(\sigma_{i \bar{k}}\right)-B_{i j}^{S} \sigma_{s \bar{k}}-B_{i \bar{k}}^{\bar{S}} \sigma_{j \bar{s}}+B_{j \bar{k}}^{\bar{s}} \sigma_{i \bar{s}}\right) \zeta^{i} \wedge \zeta^{j} \wedge \zeta^{\bar{k}},
$$

which gives that

$$
\begin{aligned}
\partial \omega & =\frac{\sqrt{-1}}{2}\left(Z_{i}\left(g_{j \bar{k}}\right)-Z_{j}\left(g_{i \bar{k}}\right)-B_{i j}^{S} g_{s \bar{k}}-B_{i \bar{k}}^{\bar{s}} g_{j \bar{s}}+B_{j \bar{k}}^{\bar{s}} g_{i \bar{s}}\right) \zeta^{i} \wedge \zeta^{j} \wedge \zeta^{\bar{k}} \\
& =\frac{\sqrt{-1}}{2} T_{i j \bar{k}} \zeta^{i} \wedge \zeta^{j} \wedge \zeta^{\bar{k}},
\end{aligned}
$$

where $\omega$ is the associated real $(1,1)$-form with respect to the almost Hermitian metric $g$.

According to the direct computation above, we may split the exterior differential operator $d: \Lambda^{p} M \otimes_{\mathbb{R}} \mathbb{C} \rightarrow$ $\Lambda^{p+1} M \otimes_{\mathbb{R}} \mathbb{C}$, into four components

$$
d=A+\partial+\bar{\partial}+\bar{A}
$$

with

$$
\begin{aligned}
\partial: \Lambda^{p, q} M \rightarrow \Lambda^{p+1, q} M, & \bar{\partial}: \Lambda^{p, q} M \rightarrow \Lambda^{p, q+1} M, \\
A: \Lambda^{p, q} M \rightarrow \Lambda^{p+2, q-1} M, & \bar{A}: \Lambda^{p, q} M \rightarrow \Lambda^{p-1, q+2} M,
\end{aligned}
$$

since we have that

$$
d\left(\Gamma\left(\Lambda^{r, s} M\right)\right) \subseteq \Gamma\left(\Lambda^{r+2, s-1} M \oplus \Lambda^{r+1, s} M \oplus \Lambda^{r, s+1} M \oplus \Lambda^{r-1, s+2} M\right) .
$$

In terms of these components, the condition $d^{2}=0$ can be written as

$$
\begin{gathered}
A^{2}=0, \quad \partial A+A \partial=0, \quad \bar{\partial} \bar{A}+\bar{A} \bar{\partial}=0, \quad \bar{A}^{2}=0, \\
A \bar{\partial}+\partial^{2}+\bar{\partial} A=0, \quad A \bar{A}+\partial \bar{\partial}+\bar{\partial} \partial+\bar{A} A=0, \quad \partial \bar{A}+\bar{\partial}^{2}+\bar{A} \partial=0 .
\end{gathered}
$$

Notice that $J$ is integrable if and only if $A=0$, equivalently, if and only if $\bar{\partial}^{2}=0$. 


\subsection{The Hodge *-operator and the adjoint operators}

We extend the inner product $\langle\cdot, \cdot\rangle$ given in (2.1), (2.2) on the bundle of real $k$-forms $\Lambda^{k} T^{\star} M$ for $1 \leq k \leq 2 n$ to the space of $(p, q)$-forms $\Lambda^{p, q} M$ defined in (2.7), $1 \leq p, q \leq n$, for $b, c \in \mathbb{C}$ and $\varphi_{i}, \psi_{i} \in \Lambda^{k} T^{\star} M, i=1$, 2, by

$$
\begin{aligned}
& \left\langle b \varphi_{1}+c \varphi_{2}, \psi\right\rangle=b\left\langle\varphi_{1}, \psi\right\rangle+c\left\langle\varphi_{2}, \psi\right\rangle, \\
& \left\langle\varphi, b \psi_{1}+c \psi_{2}\right\rangle=\bar{b}\left\langle\varphi, \psi_{1}\right\rangle+\bar{c}\left\langle\varphi, \psi_{2}\right\rangle .
\end{aligned}
$$

Locally, $(p, q)$-forms $\varphi, \psi \in \Lambda^{p, q} M$ are given by

$$
\begin{aligned}
& \varphi=\frac{1}{p ! q !} \varphi_{i_{1} \ldots i_{p} \bar{l}_{1} \ldots \bar{l}_{q}} \zeta^{i_{1}} \wedge \cdots \wedge \zeta^{i_{p}} \wedge \zeta^{\bar{l}_{1}} \wedge \cdots \wedge \zeta^{\bar{l}_{q}}, \\
& \psi=\frac{1}{p ! q !} \psi_{j_{1} \ldots j_{p} \overline{k_{1}} \ldots \overline{k_{q}}} \zeta^{j_{1}} \wedge \cdots \wedge \zeta^{j_{p}} \wedge \overline{\zeta^{\overline{k_{1}}}} \wedge \cdots \wedge \overline{\zeta^{\overline{k_{q}}}},
\end{aligned}
$$

where $\varphi_{i_{1} \ldots i_{p} \bar{l}_{1} \ldots \bar{l}_{q}}$ is skew symmetric in $i_{1}, \ldots, i_{p}$ and skew symmetric in $l_{1}, \ldots, l_{q}, \psi_{j_{1} \ldots j_{p} \bar{l}_{1} \ldots \bar{l}_{q}}$ is skew symmetric in $j_{1}, \ldots, j_{p}$ and skew symmetric in $k_{1}, \ldots, k_{q}$. Then we have that

$$
\langle\varphi, \psi\rangle=\frac{1}{p ! q !} g^{i_{1} \overline{1_{1}}} \cdots g^{i_{p} \overline{j_{p}}} g^{k_{1} \overline{l_{1}}} \cdots g^{k_{q} \overline{l_{q}}} \varphi_{i_{1} \ldots \bar{i}_{p} \overline{\bar{l}_{1}} \ldots \overline{l_{q}}} \overline{\psi_{j_{1} \ldots j_{p} \overline{k_{1}} \ldots \overline{k_{q}}}} .
$$

We define the total inner product by

$$
(\varphi, \psi):=\int_{M}\langle\varphi, \psi\rangle d V_{g},
$$

where $d V_{g}$ is the volume form defined by

$$
d V_{g}:=\frac{\omega^{n}}{n !}
$$

The Hodge * operator is the unique operator determined by the metric $g$ satisfying that for $\varphi, \psi \in \Lambda^{k} T^{\star} M$,

$$
{ }^{\star}: \Lambda^{k} T^{\star} M \rightarrow \Lambda^{2 n-k} T^{\star} M, \quad \varphi \wedge{ }^{\star} \psi=\langle\varphi, \psi\rangle d V_{g},
$$

which can be extended $\mathbb{C}$-linearly satisfying that for $\varphi, \psi \in \Lambda^{p, q} M$,

$$
\star: \Lambda^{p, q} M \rightarrow \Lambda^{n-q, n-p} M, \quad \varphi \wedge \star \bar{\psi}=\langle\varphi, \psi\rangle d V_{g} .
$$

We have that $\overline{{ }^{\star} \varphi}={ }^{\star} \bar{\varphi},{ }^{\star}{ }^{\star} \varphi=(-1)^{p+q} \varphi,\left\langle{ }^{\star} \varphi,{ }^{\star} \psi\right\rangle=\langle\varphi, \psi\rangle$. The adjoint operators $\partial^{\star}, \bar{\partial}^{\star}$ are given by

$$
(\partial \varphi, \psi)=\left(\varphi, \partial^{\star} \psi\right), \quad(\bar{\partial} \varphi, \psi)=\left(\varphi, \bar{\partial}^{\star} \psi\right) .
$$

We define that

$$
|\varphi|^{2}:=\langle\varphi, \varphi\rangle, \quad\|\varphi\|^{2}:=(\varphi, \varphi) .
$$

Lemma 2.3. Let $(M, J, \omega)$ be a compact almost Hermitian manifold. One has that

$$
\partial^{\star}=-\star \bar{\partial}^{\star}, \quad \bar{\partial}^{\star}=-\star \partial \star
$$

Proof. For $\varphi \in \Lambda^{p-1, q} M, \psi \in \Lambda^{p, q} M$, by applying the Stokes' Theorem,

$$
\begin{aligned}
\left(\varphi, \partial^{\star} \psi\right) & =(\partial \varphi, \psi) \\
& =\int_{M}\langle\partial \varphi, \psi\rangle d V_{g} \\
& =\int_{M} \partial \varphi \wedge \star \bar{\psi}
\end{aligned}
$$




$$
\begin{aligned}
& =\int_{M}(\partial+\bar{\partial}+A+\bar{A})(\varphi \wedge \star \bar{\psi})-(-1)^{p+q-1} \int_{M} \varphi \wedge \partial \star \bar{\psi} \\
& =-\int_{M} \varphi \wedge(-1)^{p+q-1} \partial \star \bar{\psi} \\
& =-\int_{M} \varphi \wedge^{\star \star} \partial^{\star} \bar{\psi} \\
& \left.=\int_{M} \varphi \wedge^{\star\left(\overline{-{ }^{\star} \bar{\partial}} \psi\right.}\right),
\end{aligned}
$$

where we used that $\bar{\partial}\left(\varphi \wedge^{\star} \bar{\psi}\right)=A\left(\varphi \wedge^{\star} \bar{\psi}\right)=\bar{A}\left(\varphi \wedge^{\star} \bar{\psi}\right)=0$ since $\varphi \wedge^{\star} \bar{\psi} \in \Lambda^{n-1, n} M$.

We write $\iota_{i} \varphi:=\iota_{Z_{i}} \varphi, \iota_{\bar{j}} \varphi:=\iota_{Z_{\bar{j}}} \varphi$. It follows from (2.5),

$$
\left\langle\varphi, \zeta^{i} \wedge \psi\right\rangle=\left\langle g^{j \bar{i}}{ }_{l j} \varphi, \psi\right\rangle, \quad\left\langle\varphi, \zeta^{\bar{i}} \wedge \psi\right\rangle=\left\langle g^{i \bar{j}}{ }_{l} \varphi, \psi\right\rangle .
$$

We define the Lefschetz operator $L: \Lambda^{p, q} M \rightarrow \Lambda^{p+1, q+1} M$ and its adjoint operator $\Lambda: \Lambda^{p+1, q+1} M \rightarrow \Lambda^{p, q} M$ by

$$
L \varphi=\omega \wedge \varphi, \quad\langle L \varphi, \psi\rangle=\langle\varphi, \Lambda \psi\rangle .
$$

Locally, we obtain that from (2.20),

$$
\Lambda=\sqrt{-1} g^{i \bar{j}} l_{i} l_{j} .
$$

For a $(p, q)$-form $\varphi \in \Lambda^{p, q} M$ with $p+q=k \leq n$, we have that $[L, \Lambda] \varphi=(k-n) \varphi$. Applying this repeatedly, we obtain that

$$
\left[L^{r}, \Lambda\right] \varphi=\left[L^{r-s}, \Lambda\right] L^{s} \varphi+s(k-n+s-1) L^{r-1} \varphi .
$$

Especially, we have that for $s=r$,

$$
\left[L^{r}, \Lambda\right] \varphi=r(k-n+r-1) L^{r-1} \varphi
$$

Definition 2.1. We call a $(p, q)$-form $\varphi$ primitive if $\Lambda \varphi=0$.

For a primitive $(p, q)$-form $\varphi$ with $p+q=k \leq n$, we have that

$$
\Lambda(\omega \wedge \varphi)=(n-k) \varphi
$$

and

$$
\Lambda L^{r} \varphi=r(n-k-r+1) L^{r-1} \varphi
$$

\section{Proof of Theorem 1.1 and some Corollaries}

Proof of Theorem 1.1. Let $\left(M^{2 n}, J, \omega\right)$ be an almost Hermitian manifold of real dimension $2 n$ with $n \geq 3$. Let $g$ be the almost Hermitian metric associated to the real $(1,1)$-form $\omega$. We define the torsion $(1,0)$-form (cf. [17]) on $M$ by

$$
w_{i}:=T_{i k}^{k}=g^{k \bar{l}} T_{i k \bar{l}}, \quad \eta:=-w_{i} \zeta^{i} .
$$

Then we have from (2.4), (2.17) and (2.21),

$$
\eta=-\Lambda \partial \omega
$$

Lemma 3.1. Let $\left(M^{2 n}, J, \omega\right)$ be an almost Hermitian manifold with $n \geq 3$. Then one has that

$$
\partial \omega^{n-1}=-\eta \wedge \omega^{n-1} .
$$


Proof. Since we have that $\Lambda\left(\partial \omega-\frac{1}{n-1} L(\Lambda \partial \omega)\right)=0$ from (2.24) for $r=k=1$, and also since we have that $\left[L^{n-k+1}, \Lambda\right] \varphi=0$ for $\varphi \in \Lambda^{p, q} M$ with $p+q=k \leq n$ from (2.22), we obtain that $\Lambda L^{n-2}\left(\partial \omega-\frac{1}{n-1} L(\Lambda \partial \omega)\right)=0$. Then, since $\Lambda$ is injective, we obtain that (cf. [16, Lemma 6.24]),

$$
L^{n-2}\left(\partial \omega-\frac{1}{n-1} L(\Lambda \partial \omega)\right)=0
$$

which gives us that from (3.1),

$$
\partial \omega^{n-1}=L(\Lambda \partial \omega) \wedge \omega^{n-2}=-\eta \wedge \omega^{n-1} .
$$

Lemma 3.2. Let $\left(M^{2 n}, J, \omega\right)$ be a compact almost Hermitian manifold with $n \geq 3$. Then one has that

$$
\eta=-\Lambda \partial \omega=\sqrt{-1}^{\star} \omega
$$

Proof. For $\varphi \in \Lambda^{1,0} M$, by applying Lemma 2.3 and (3.2), and using $\star \omega=\frac{\omega^{n-1}}{(n-1) !}$,

$$
\begin{aligned}
\left\langle\varphi, \bar{\partial}^{\star} \omega\right\rangle \frac{\omega^{n}}{n !} & =\left\langle\varphi,{ }^{\star} \partial\left(\frac{1}{(n-1) !} \omega^{n-1}\right)\right\rangle \frac{\omega^{n}}{n !} \\
& =\left\langle\varphi,{ }^{\star}\left(\frac{1}{(n-1) !} \eta \wedge \omega^{n-1}\right)\right\rangle \frac{\omega^{n}}{n !} \\
& =\varphi \wedge{ }^{\star}\left(\frac{1}{(n-1) !} \bar{\eta} \wedge \omega^{n-1}\right) \\
& =-\varphi \wedge \bar{\eta} \wedge \frac{\omega^{n-1}}{(n-1) !} \\
& =-\langle\varphi \wedge \bar{\eta}, \omega\rangle \frac{\omega^{n}}{n !} \\
& =-\langle\varphi, \sqrt{-1} \eta\rangle \frac{\omega^{n}}{n !},
\end{aligned}
$$

which gives the desired equality.

From Lemma 3.1, we have the following equivalence.

Lemma 3.3. On an almost Hermitian manifold $\left(M^{2 n}, J, \omega\right)$ with $n \geq 3$, the metric $\omega$ is semi-Kähler if and only if the torsion (1,0)-form $\eta$ satisfies $\eta=0$.

Proof. Since we have $d\left(\omega^{n-1}\right)=(\partial+\bar{\partial}+A+\bar{A})\left(\omega^{n-1}\right)=(\partial+\bar{\partial})\left(\omega^{n-1}\right)$, where we used that $A\left(\omega^{n-1}\right)=\bar{A}\left(\omega^{n-1}\right)=$ 0 , the semi-Kählerity $d\left(\omega^{n-1}\right)=0$ is equivalent to that $\partial\left(\omega^{n-1}\right)=\bar{\partial}\left(\omega^{n-1}\right)=0$. From Lemma 3.1, $\partial\left(\omega^{n-1}\right)=0$ and $\bar{\partial}\left(\omega^{n-1}\right)=0$ are equivalent to that $\eta=0$ and $\bar{\eta}=0$ respectively.

We say that the torsion $(1,0)$-form $\eta$ is holomorphic when it satisfies that $\bar{\partial} \eta=0$.

Corollary 3.1. Let $\left(M^{2 n}, J, \omega\right)$ be a compact almost Hermitian manifold with $n \geq 3$. If the torsion $(1,0)$-form $\eta$ is holomorphic, then $\omega$ is semi-Kähler.

Proof. Assuming that $\eta$ is holomorphic, we obtain that $0=\bar{\partial} \eta=\sqrt{-1} \bar{\partial} \bar{\partial}^{\star} \omega$ by applying the formula (3.3). Then we have that $\left\|\bar{\partial}^{\star} \omega\right\|^{2}=\left(\bar{\partial} \bar{\partial}^{\star} \omega, \omega\right)=0$. Hence, we obtain that $\eta=\sqrt{-1} \bar{\partial}^{\star} \omega=0$, which implies that $\omega$ is semi-Kähler from Lemma 3.3.

We can obtain the following lemma in almost Hermitian geometry as well.

Lemma 3.4. (cf. [1, Lemma 2.6]) Let $\left(M^{2 n}, J, \omega\right)$ be a compact almost Hermitian manifold with $n \geq 3$ and let $\tau \in \Lambda^{1,0} M$. Then one has that

$$
\partial^{\star} \tau=\langle\sqrt{-1} \bar{\partial} \tau, \omega\rangle+\left\langle\tau, \sqrt{-1} \bar{\partial}^{\star} \omega\right\rangle .
$$


Proof. As in [1], we compute that

$$
\varphi \wedge^{\star} \bar{\tau}=\langle\varphi, \tau\rangle d V_{g}=\sqrt{-1}\langle\varphi \wedge \bar{\tau}, \omega\rangle d V_{g}=\varphi \wedge\left(\overline{\left(-\sqrt{-1} \tau \wedge \frac{\omega^{n-1}}{(n-1) !}\right.}\right),
$$

which gives us that ${ }^{\star} \tau=-\sqrt{-1} \tau \wedge \frac{\omega^{n-1}}{(n-1) !}$. Then, by applying Lemma 2.3 and (3.2), we obtain that

$$
\begin{aligned}
\partial^{\star} \tau & =-{ }^{\star} \bar{\partial} \star \\
& =\star \bar{\partial}\left(\sqrt{-1} \tau \wedge \frac{\omega^{n-1}}{(n-1) !}\right) \\
& =\star\left(\sqrt{-1} \bar{\partial} \tau \wedge \frac{\omega^{n-1}}{(n-1) !}+\sqrt{-1} \tau \wedge \bar{\eta} \wedge \frac{\omega^{n-1}}{(n-1) !}\right) \\
& =\star\left(\langle\sqrt{-1} \bar{\partial} \tau, \omega\rangle d V_{g}+\sqrt{-1}\langle\tau \wedge \bar{\eta}, \omega\rangle d V_{g}\right) \\
& =\langle\sqrt{-1} \bar{\partial} \tau, \omega\rangle+\left\langle\tau, \sqrt{-1} \bar{\partial}^{\star} \omega\right\rangle,
\end{aligned}
$$

where we used that ${ }^{\star} \omega=\frac{\omega^{n-1}}{(n-1) !}, \bar{\partial} \omega^{n-1}=-\bar{\eta} \wedge \omega^{n-1},\langle\tau \wedge \bar{\eta}, \omega\rangle=\langle\tau, \sqrt{-1} \eta\rangle=-\sqrt{-1}\langle\tau, \eta\rangle$ and $\eta=\sqrt{-1} \bar{\partial}^{\star} \omega$.

Proposition 3.1. Let $\left(M^{2 n}, J, \omega\right)$ be a compact almost Hermitian manifold with $n \geq 3$. Then one has that

$$
s-\hat{s}=-\partial^{\star} \eta+|\eta|^{2}+B_{i j}^{\bar{r}} B_{\bar{r} \bar{j}}^{i},
$$

where $s$ is the Chern scalar curvature and $\hat{s}$ is a Riemannian type scalar curvature of the metric $\omega$ with respect to the Chern connection in (2.11). Notice that $B_{i j}^{\bar{r}} B_{\bar{r} \bar{j}}^{i}$ means that we sum over repeated indices $i, j$ and $r$ with respect to the metric $\omega$.

Proof. We compute that from (2.15),

$$
\begin{aligned}
\bar{\partial}^{\star} \bar{\partial}^{\star} \omega & =\sqrt{-1} \partial_{\bar{j}} w_{i} \zeta^{\bar{j}} \wedge \zeta^{i} \\
& =-\sqrt{-1} \nabla_{\bar{j}} w_{i} \zeta^{i} \wedge \zeta^{\bar{j}} \\
& =-\sqrt{-1} \bar{\partial} \eta,
\end{aligned}
$$

where $\nabla$ is the Chern connection.

On the other hand, we have from (2.12),

$$
P_{i \bar{j}}-g^{k \bar{l}} R_{k \bar{j} \bar{l} \bar{l}}=\nabla_{\bar{j}} T_{s i}^{S}-B_{i s}^{\bar{r}} B_{\bar{\gamma} \bar{j}}^{S}=-\nabla_{\bar{j}} w_{i}+B_{s i}^{\bar{r}} B_{\bar{r} \bar{j}}^{S},
$$

where we used that $T_{s i}^{S}=-T_{i s}^{S}=-w_{i}$ and $B_{i s}^{\bar{r}}=-B_{s i}^{\bar{r}}$. By combining (3.5) with (3.6), and by summing over indices $i, j$ with respect to the metric $\omega$, we obtain that

$$
s-\hat{s}=\left\langle\bar{\partial} \bar{\partial}^{\star} \omega, \omega\right\rangle+B_{S i}^{\bar{r}} B_{\bar{i}}^{S} .
$$

By applying Lemma 3.4 for $\tau=\eta$, we obtain

$$
\begin{aligned}
\partial^{*} \eta & =\langle\sqrt{-1} \bar{\partial} \eta, \omega\rangle+\left\langle\eta, \sqrt{-1}^{\star} \omega\right\rangle \\
& =-\left\langle\bar{\partial} \bar{\partial}^{\star} \omega, \omega\right\rangle+|\eta|^{2},
\end{aligned}
$$

where we used that $\eta=\sqrt{-1} \bar{\partial}^{\star} \omega$. By plugging (3.8) into (3.7), we obtain the desired formula (3.4).

We compute directly that $\partial \bar{\partial} \omega^{k} \wedge \omega^{n-k-1}=k \partial \bar{\partial} \omega \wedge \omega^{n-2}+k(k-1) \partial \omega \wedge \bar{\partial} \omega \wedge \omega^{n-3}, \frac{k}{n-1} \partial \bar{\partial} \omega^{n-1}=k \partial \bar{\partial} \omega \wedge$ $\omega^{n-2}+k(n-2) \partial \omega \wedge \bar{\partial} \omega \wedge \omega^{n-3}$. Combining these we obtain that

$$
\partial \bar{\partial} \omega^{k} \wedge \omega^{n-k-1}=\frac{k}{n-1} \partial \bar{\partial} \omega^{n-1}-k(n-k-1) \partial \omega \wedge \bar{\partial} \omega \wedge \omega^{n-3} .
$$

Lemma 3.5. Let $\left(M^{2 n}, J, \omega\right)$ be a compact almost Hermitian manifold with $n \geq 3$. Then one has that

$$
\star\left(\sqrt{-1} \partial \bar{\partial} \omega^{n-1}\right)=(n-1) !\left(\hat{s}-s+\left|\partial^{\star} \omega\right|^{2}+B_{i j}^{\bar{r}} B_{\bar{r} \bar{j}}^{i}\right),
$$

which tells us that the metric $\omega$ is Gauduchon if and only if $s-\hat{s}=\left|\partial^{\star} \omega\right|^{2}+B_{i j}^{\bar{r}} B_{\bar{r} \bar{j}}^{i}$. 
Proof. Note that we have from (2.19),

$$
\partial \bar{\partial} \omega^{n-1}=-(\bar{\partial} \partial+A \bar{A}+\bar{A} A) \omega^{n-1}=-\bar{\partial} \partial \omega^{n-1}
$$

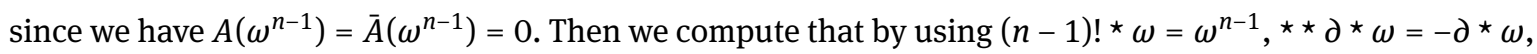
Lemma 2.3 and Proposition 3.1,

$$
\begin{aligned}
\sqrt{-1} \star \partial \bar{\partial} \omega^{n-1} & =-\sqrt{-1} \star \bar{\partial} \partial \omega^{n-1} \\
& =\sqrt{-1}(n-1) ! \star \bar{\partial} \star \star \partial \star \omega \\
& =(n-1) ! \partial^{\star}\left(\sqrt{-1} \bar{\partial}^{\star} \omega\right) \\
& =(n-1) ! \partial^{\star} \eta \\
& =(n-1) !\left(\hat{s}-s+|\eta|^{2}+B_{i j}^{\bar{r}} B_{\bar{r} \bar{j}}^{i}\right),
\end{aligned}
$$

which gives the desired equality by combining (3.10) with $\bar{\eta}=-\sqrt{-1} \partial^{\star} \omega$.

Lemma 3.6. (cf. [1, Lemma 4.2]) Let $\left(M^{2 n}, J, \omega\right)$ be a compact almost Hermitian manifold with $n \geq 3$. Then one has that

$$
\star\left(\sqrt{-1} \partial \omega \wedge \bar{\partial} \omega \wedge \omega^{n-3}\right)=(n-3) !\left(\left|\partial^{\star} \omega\right|^{2}-|\partial \omega|^{2}\right) .
$$

Proof. We can show the equality of [16, Proposition 6.29] by replacing $\left\{d z_{i}\right\}$ with a local coframe $\left\{\zeta^{i}\right\}$ associated to a local unitary frame $\left\{Z_{i}\right\}$ with respect to the almost Hermitian metric $g$. Note that unitary frames always exist locally since we can take any frame and apply the Gram-Schmidt process. Then with respect to a local unitary frame with respect to $g$, we have $g_{i \bar{j}}=\delta_{i j}$ for any $i, j=1, \ldots, n$ and the associated real $(1,1)$-form $\omega$ with respect to $g$ takes the local expression $\omega=\sqrt{-1} \sum_{i=1}^{n} \zeta^{i} \wedge \zeta^{\bar{i}}$. Applying the equality of [16, Proposition 6.29], we have for a primitive $\alpha \in \Lambda^{p, q} M$ with $p+q=k \leq n$,

$$
{ }^{\star} \alpha=(-1)^{p}(\sqrt{-1})^{k^{2}} \frac{L^{n-k} \alpha}{(n-k) !},
$$

Since $\partial \omega+\frac{\sqrt{-1}}{n-1} L \bar{\partial}^{\star} \omega$ is primitive, we obtain that

$$
\star \partial \omega=\sqrt{-1} \partial \omega \wedge \frac{\omega^{n-3}}{(n-3) !}-\frac{n-2}{(n-1) !} \bar{\partial}^{\star} \omega \wedge \omega^{n-2}-\frac{\sqrt{-1}}{n-1} \star L \bar{\partial}^{\star} \omega .
$$

From the definition of the operators $L, \Lambda$, for any $\varphi \in \Lambda^{p-1, q-1} M$ and $\psi \in \Lambda^{p, q} M$,

$$
\langle L \varphi, \psi\rangle d V_{g}=\varphi \wedge{ }^{\star \star-1} L^{\star} \psi=\left\langle\varphi,{ }^{\star-1} L^{\star} \psi\right\rangle d V_{g},
$$

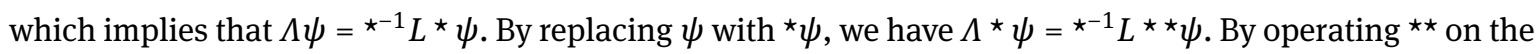
both side, we have ${ }^{\star \star} \Lambda^{\star} \psi=(-1)^{p+q \star} L \psi$. Since we have $\Lambda^{\star} \psi \in \Lambda^{n-q-1, n-p-1} M$ and ${ }^{\star \star} \Lambda{ }^{\star} \psi=(-1)^{-(p+q)} \Lambda^{\star} \psi$, we obtain that $\Lambda^{\star} \psi={ }^{\star} L \psi$. By combining (3.11) with ${ }^{\star} L=\Lambda^{\star}$, we get that

$$
\frac{\sqrt{-1}}{n-1} \star L \bar{\partial}^{\star} \omega=\bar{\partial}^{\star} \omega \wedge \frac{\omega^{n-2}}{(n-1) !},
$$

which gives that

$$
\star \partial \omega=\sqrt{-1} \partial \omega \wedge \frac{\omega^{n-3}}{(n-3) !}-\bar{\partial}^{\star} \omega \wedge \frac{\omega^{n-2}}{(n-2) !} .
$$

From Lemma 2.2, we have

$$
\star \bar{\partial}^{\star} \omega=-\star \star \partial \star \omega=-\star \star \partial\left(\frac{\omega^{n-1}}{(n-1) !}\right)=\frac{\partial \omega^{n-1}}{(n-1) !} .
$$

Then we obtain that by combining (3.12) with (3.13),

$$
\partial \omega \wedge \star \overline{\partial \omega}=-\sqrt{-1} \partial \omega \wedge \bar{\partial} \omega \wedge \frac{\omega^{n-3}}{(n-3) !}+\partial^{\star} \omega \wedge \star \overline{\partial^{\star} \omega},
$$

which gives the desired equality. 
Combining Lemma 3.5, 3.6 with (3.9), we obtain the following.

Lemma 3.7. Let $\left(M^{2 n}, J, \omega\right)$ be a compact almost Hermitian manifold with $n \geq 3$ and let $k$ be an integer such that $1 \leq k \leq n-1$. Then one has that

$$
\star\left(\sqrt{-1} \partial \bar{\partial} \omega^{k} \wedge \omega^{n-k-1}\right)=k(n-2) !\left(\frac{k-1}{n-2}\left|\partial^{\star} \omega\right|^{2}+\frac{n-k-1}{n-2}|\partial \omega|^{2}-s+\hat{s}+B_{i j}^{\bar{r}} B_{\bar{r} \bar{j}}^{i}\right) .
$$

Lemma 3.7 implies the equivalence in Theorem 1.1.

Proof of Corollary 1.1. By combining Lemma 3.3 with Proposition 3.1, we conclude that if a compact almost Hermitian manifold is semi-Kähler, since then we have that $\eta=0$, we obtain that

$$
s-\hat{s}=-\partial^{\star} \eta+|\eta|^{2}+B_{i j}^{\bar{r}} B_{\bar{r} \bar{j}}^{i}=B_{i j}^{\bar{r}} B_{\bar{r} \bar{j}}^{i} .
$$

If additionally, it is also $k$-th Gauduchon for an integer $k$ such that $1 \leq k \leq n-2$, from the equivalence in Theorem 1.1, we obtain that since we have that $\partial^{\star} \omega=\sqrt{-1} \bar{\eta}=0$,

$$
s-\hat{s}=\frac{n-k-1}{n-2}|\partial \omega|^{2}+B_{i j}^{\bar{r}} B_{\bar{r} \bar{j}}^{i} .
$$

By combining (3.14) with (3.15) for $1 \leq k \leq n-2$, we must have that $\partial \omega=0$, which tells us that the metric $\omega$ is quasi-Kähler.

By combining Corollary 1.1 with Corollary 3.1, we obtain the following result.

Corollary 3.2. Let $\left(M^{2 n}, J, \omega\right)$ be a compact $k$-th Gauduchon almost Hermitian manifold with $n \geq 3$ and let $k$ be an integer such that $1 \leq k \leq n-2$. If the torsion $(1,0)$-form $\eta$ is holomorphic on $M$, then $\omega$ is quasi-Kähler.

By combining Corollary 1.2 with Corollary 3.1, we obtain the following result.

Corollary 3.3. Let $\left(M^{2 n}, J, \omega\right)$ be a compact almost pluriclosed manifold with $n \geq 3$. If the torsion $(1,0)$-form $\eta$ is holomorphic on $M$, then $\omega$ is quasi-Kähler.

The following equivalence can be obtained from Theorem 1.1 and Lemma 3.5, 3.6.

Corollary 3.4. Let $\left(M^{2 n}, J, \omega\right)$ be a compact almost Hermitian manifold with $n \geq 3$ and let $k$ be an integer such that $1 \leq k \leq n-1$. Then the following are equivalent:

(i) $\omega$ is $k$-th Gauduchon for all $k$ such that $1 \leq k \leq n-1$;

(ii) $|\partial \omega|^{2}=\left|\partial^{\star} \omega\right|^{2}=\left\langle\bar{\partial} \bar{\partial}^{\star} \omega, \omega\right\rangle$.

Proof. Assume that $\omega$ is $k$-th Gauduchon for all $1 \leq k \leq n-1$. Then, since especially the metric $\omega$ is Gauduchon, and since we have that from (3.9),

$$
0=\partial \bar{\partial} \omega^{n-1}=(n-1)(n-k-1) \partial \omega \wedge \bar{\partial} \omega \wedge \omega^{n-3}
$$

for all $1 \leq k \leq n-1$, by applying Lemma 3.6, we obtain that $|\partial \omega|^{2}=\left|\partial^{\star} \omega\right|^{2}$. Then, from the equivalence in Theorem 1.1, we have that $s-\hat{s}=\left|\partial^{\star} \omega\right|^{2}+B_{i j}^{\bar{r}} B_{\bar{r} \bar{j}^{j}}^{i}$. Combining with the formula (3.7), we obtain that $\left|\partial^{\star} \omega\right|^{2}=$ $\left\langle\bar{\partial} \bar{\partial}^{\star} \omega, \omega\right\rangle$.

On the other hand, assuming that $|\partial \omega|^{2}=\left|\partial^{\star} \omega\right|^{2}=\left\langle\bar{\partial} \bar{\partial}^{\star} \omega, \omega\right\rangle$, then we get that from the formula (3.7), $s-\hat{s}=\left|\partial^{\star} \omega\right|^{2}+B_{i j}^{\bar{r}} B_{\bar{r} \bar{j}}^{i}$, which is equivalent to that the metric $\omega$ is Gauduchon from the equivalence in Lemma 3.5. Additionally, in this case, by applying Lemma 3.6, we have that $\partial \omega \wedge \bar{\partial} \omega \wedge \omega^{n-3}=0$, which gives us that from (3.9),

$$
0=\frac{k}{n-1} \partial \bar{\partial} \omega^{n-1}=\partial \bar{\partial} \omega^{k} \wedge \omega^{n-k-1}
$$

for all $1 \leq k \leq n-1$. 
We introduce the following result in [10].

Proposition 3.2. (cf. [10, Corollary 1.1]) Let $\left(M^{2 n}, J, \omega\right)$ be compact almost Hermitian manifold with $n \geq 3$. Assume that $\omega$ satisfies that for all $1 \leq k \leq n-2$,

$$
\frac{\sqrt{-1}}{2} \partial \bar{\partial} \omega^{k} \wedge \omega^{n-k-1}=C_{\omega, k} \omega^{n}
$$

for some constant $C_{\omega, k} \in \mathbb{R}$. If the metric $\omega$ is almost pluriclosed, then $\omega$ is $k$-th almost Gauduchon for any $1 \leq k \leq n-1$.

Combining Corollary 3.4 with Proposition 3.2, we obtain the following result.

Corollary 3.5. Let $\left(M^{2 n}, J, \omega\right)$ be compact almost Hermitian manifold with $n \geq 3$. Assume that $\omega$ satisfies that for all $1 \leq k \leq n-2$,

$$
\frac{\sqrt{-1}}{2} \partial \bar{\partial} \omega^{k} \wedge \omega^{n-k-1}=C_{\omega, k} \omega^{n}
$$

for some constant $C_{\omega, k} \in \mathbb{R}$. If the metric $\omega$ is almost pluriclosed, then one has that $|\partial \omega|^{2}=\left|\partial^{\star} \omega\right|^{2}=$ $\left\langle\bar{\partial} \bar{\partial}^{\star} \omega, \omega\right\rangle$.

Conflict of interest: Authors state no conflict of interest.

\section{References}

[1] Chen, H., Chen, L. and Nie, X. Chern-Ricci curvatures, holomorphic sectional curvature and Hermitian metrics, Sci. China Math. 64 (2021), 763-780.

[2] Fino, A., Ugarte, L. On generalized Gauduchon metrics, Proc. Edinb. Math. Soc. 56 (2013), 733-753.

[3] Fu, J., Wang, Z. and Wu, D. Semilinear equations, the $\gamma_{k}$ function, and generalized Gauduchon metrics, J. Eur. Math. Soc. 15 (2013), 659-680.

[4] Gauduchon, P. Le thèorème de l'excentricité nulle, C. R. Acad. Sci. Paris Sér. A-B 285 (1977), no. 5, A387-A390.

[5] Gauduchon, P. Hermitian connections and Dirac operators, Boll. Un. Mat. Ital. B (7) (suppl.) 11 (2) (1997), 257-288.

[6] Gray, A. Some examples of almost Hermitian manifolds, Illinois J. Math. 10 (1966), 353-366.

[7] Hsiung, C C. Almost complex and complex structures, World Scientific, Series in Pure Mathematics-Volume 20, (1995), ISBN 981-02-1712-9.

[8] Jost, J., Yau, S.-T. A non-linear elliptic system for maps from Hermitian to Riemannian manifolds and rigidity theorems in Hermitian geometry, Acta Math. 170 (1993), 221-254.

[9] Kawamura, M. On Kähler-like and G-Kähler-like almost Hermitian manifolds, Complex Manifolds 7 (2020) 145-161.

[10] Kawamura, M. On the $k$-th almost Gauduchon condition on almost Hermitian manifolds, Analysis (2021). https://doi.org/10.1515/anly-2021-0021

[11] Kawamura, M. On the conformally $k$-th almost Gauduchon condition and the conformally almost balanced condition, Cubo, A Mathematical Journal 23 (2021), no.2, 333-341.

[12] Latorre, A., Ugarte, L. On non-Kähler compact complex manifolds with balanced and astheno-Kähler metrics, C. R. Acad. Sci. Paris, Ser. 1335 (2017), 90-93.

[13] Liu, K., Yang, X. Ricci curvatures on Hermitian manifolds, Trans. Amer. Math. Soc. 369 (2017), 5157-5196.

[14] Popovici, D. Aeppli cohomology classes associated with Gauduchon metrics on compact complex manifolds, Bulletin de la Société Mathématique de France, 143 (2015), no. 4, 763-800.

[15] Vezzoni, L. On Hermitian curvature flow on almost complex manifolds, Diff. Geom. and its Appl. 29 (2011), 709-722.

[16] Voisin, C. Hodge theory and complex Algebraic geometry I,Cambridge Studies in Advanced Mathematics, 76 Cambridge University Press, Cambridge, (2002), $\mathrm{x}+322$ pp. ISBN: 0-521-80260-1.

[17] Yang, B. and Zheng, F. On curvature tensors of Hermitian manifolds, Comm. Anal. Geom. (5) 26 (2018), 1195-1222.

[18] Zheng, T. An almost complex Chern-Ricci flow, J. Geom. Anal. 28(2018), 2129-2165. 